\title{
Heart rate variability and heart rate under general anesthesia in rats of both sexes
}

\author{
P. Svorc ${ }^{1,2 *}$, D. Petrasova ${ }^{3}$ and P. Svorc Jr. ${ }^{2}$ \\ ${ }^{1}$ Department of Physiology, Medical Faculty Safarik's University, Kosice, Slovak Republic \\ ${ }^{2}$ Department of Physiology and Pathophysiology, Medical Faculty Ostrava University, Ostrava, Czech Republic \\ ${ }^{3}$ Laboratory of Research Bio-models, Medical Faculty Safarik's University, Kosice, Slovak Republic
}

\begin{abstract}
Introduction: The primary objective of this study was to determine the impact of spectral powers of heart rate variability (HRV) on changes in heart rate (HR), total spectral power of HRV, and low-frequency (LF)/high-frequncy (HF) ratio in healthy, sexually mature rats of both sexes spontaneously breathing under zoletil anesthesia in the light (inactive) and the dark (active) period of their regimen day.

Material and methods: Experiments were performed using male and female zoletil-anesthetized ( $30 \mathrm{mg} / \mathrm{kg}$ [intraperitoneal]) Wistar rats after a four-week adaptation to a light-dark (LD) cycle (12h:12h). The animals were divided into four experimental groups ( $\mathrm{n}=20$ each) according to sex and light period. HR, spectral powers of $\mathrm{HRV}$ (very low frequency, LF, and HF), as well as LF/HF ratio were evaluated $20 \mathrm{~min}$ after administration of anesthesia.

Results and conclusions: Zoletil exerted a tachycardic effect in both sexes and in both light periods of the regimen day. In females, the autonomic nervous system was involved in HR changes in both light periods, while in males, HR exhibited no dependence on autonomic nervous system activity; as such, the authors speculate that it was predominantly determined by other factors. In females, HRV was determined by sympathetic and baroreflex activity in both light periods, while in males, HRV was determined by parasympathetic activity. LF significantly influenced LF/HF ratio in females, but not in males, while the effect of $\mathrm{HF}$ on the LF/HF ratio was negligible in both sexes and in both light periods.
\end{abstract}

\section{Introduction}

General anesthesia is known to potentiate parasympathetic activity and weaken sympathetic and baroreflex activity [1]. These effects should be avoided as much as possible because it limits a subject's ability to respond to physiological perturbations during surgery [2]. Therefore, the choice of anesthetic is very important and, no less important, is to know to what extent the tone of the autonomic nervous system (ANS) is affected under general anesthesia and the potential impact on the cardiovascular system, which is primarily under ANS control.

Heart rate (HR) is the result of mutual interactions between vagal and sympathetic activity. In this regard, the use of the HR variability (HRV) method can be an effective tool in evaluating autonomic heart control, in which changes in HRV are a useful indicator of tendencies of heart rhythm disorders.

However, there are currently relatively little data describing changes in ANS activity during general anesthesia as well as possible sex differences. A concept that sex-based variations should also be taken into account has been proposed because different cardiovascular regulation has been described in females [3]. Koresh et al. [4] referred to this phenomenon, in which male rats exhibit significantly higher heart rhythm and lower HRV parameters than females in the both active as well as non-active periods of the rat regimen day. However, there is no supportive evidence of changes in HRV in rats over a $24 \mathrm{~h}$ period.

Zoletil anesthesia is used by veterinarians mainly in domestic animals and those living in the wild, but is not commonly used in experiments. It is reported that this type of anesthesia is suitable because it does not affect vital functions. However, the unanswered question is whether there are sex differences depending on the time of day general anesthesia is administered. The primary objective of this study was to determine the impact of spectral powers of HRV on changes in HR, on total spectral power of HRV and on low-frequency (LF)/high-frequency (HF) ratio in healthy, sexually mature rats of both sexes, spontaneously breathing under zoletil anesthesia in the light (inactive) and the dark (active) period of their regimen day.

\section{Materials and methods}

The present study conformed to the Guide for the Care and Use of Laboratory Animals published by the United States National Institutes of Health (publication number 85-23, revised 1996). The study protocol was approved by the Ethics Committee of the Medical Faculty of Safarik University (Kosice, Slovak Republic; permission number 2/05 and permission number ŠVPS SR: Ro-4234/15-221).

The experiments were performed using zoletil-anesthetized (Zoletil, $30 \mathrm{mg} / \mathrm{kg}$, Virbac, France) Wistar rats (mean weight, $340 \pm 40$ g, 3-4 months of age) and after adaptation to a light-dark (LD) cycle (12h light:12h dark) for four weeks. The rats were fed a standard pellet

${ }^{*}$ Correspondence to: P. Svorc, Department of Physiology, Medical Faculty Safarik’s University, Kosice, Slovak Republic, E-mail: pavol.svorc@upjs.sk

Key words: chronobiology, heart rate variability, anesthesia, sex differences, rat

Received: September 09, 2020; Accepted: September 21, 2020; Published: September 23, 2020 
diet, with ad libitum access to food and water. The depth of anesthesia was assessed according to whether a painful stimulus caused noticeable motor movements (minimal limb movement, muscle tension change) or cardiovascular responses, such as change in HR or onset of heart rhythm disorders. Animal handling was performed by professional staff at the menagerie. Animals were randomly divided into four experimental groups $(\mathrm{n}=20$ each) according to sex and light conditions.

HRV was analyzed using the ID Instruments computer system for biopotential recording from an average of 220 heart cycles, 20 min after administration of anesthesia. The focus was on the assessment of the impact of the spectral powers of HRV on changes in HR, total spectral power of HRV, and LF/HF ratio.

Correlations were calculated as a correlation coefficients, in which the coefficient was statistically significant in the range of -0.4 to -1 , and from +0.4 to +1 . The experiments were conducted throughout the year and the results were averaged independent of season and, in females, independent of estral cycle.

\section{Results and discussion}

\section{HR}

In the present study, we focused on sex differences in HR during the active and nonactive periods of the rat regimen day (what may be considered parallel to circadian rhythm). In females, LD differences in HR (light $441 \pm 61$ versus [vs.] dark $446 \pm 29$ beats $/ \mathrm{min}$ ) were eliminated, unlike in males (light $415 \pm 24$ vs. dark $482 \pm 19$ beats/ $\mathrm{min})$, in which they differed significantly $(\mathrm{p}<0.001)$. Significant sex differences $(p<0.001)$ were observed only in the dark period with higher values in males.

Because HR in non-anesthetized rats exhibits circadian variation; in the dark period, it varies from 347 to 363 beats/min and, in the light period from 309 to 321 beats/min. Molcan et al. [5] reported that recorded HR was increased in both sexes and in both light periods. In females, LD differences were eliminated (possibly modified) and, in males, LD differences were preserved. Based on this result, we speculate that males probably respond diffferently to general anesthesia than females with regard to maintenance of circadian rhythms in HR.

Based on our results, however, the question emerged that if tachycardia in both sexes and both light periods of the regimen day was detected during a period dominated by parasympathetic activity, what is the impact of spectral powers and the total spectral power of HRV on changes in HR? To answer this question, we used correlation coefficients calculated for the spectral forces of VLF, LF, HF, and HR (Table 1), which revealed sex differences. In females, changes in HR were significantly dependent on ANS activity in both light periods, in which parasympathetic activity was more prominent. A negative correlation could, in part, explain the increase in HR. Similar results were also obtained for total spectral power of HRV and HR (Table 1). In males, changes in HR demonstrated no dependence on ANS activity in both light periods; therefore, we assume that it was predominantly determined by other factors.
Thus, a paradox emerges in which, on one hand, there is clear evidence of increased parasympathetic activity and, on the other, increased HR. This paradox has also been described in dogs under inhalation anesthesia, in which it is assumed that different anesthetics have a different impact on vagolytic activity [6]. Such an explanation is described in the review by Yuan and Silberstein [7] regarding the vagal nerve and its stimulation, which is currently undergoing many trials to investigate their potential for various clinical disorders. Although this is an example of bronchodilation after stimulation of the vagal nerve, the authors allude to experimental work in guinea pigs, in which lowvoltage stimulation of the vagal nerve caused a moderate increase in plasma levels of adrenaline and noradrenaline.

\section{Total power of HRV}

Total spectral power of HRV reflects overall autonomic activity. The total spectral power of HRV demonstrated sex differences in both light periods. However, what is the proportion of VLF, LF and HF in the total power of HRV? The changes in HRV in both sexes depended on all spectral powers of HRV in both light periods. It appears that in females, the proportion of VLF and LF are more pronounced than HF, while in males, HF predominates in both light periods (Table 2).

\section{LF/HF ratio}

The LF/HF ratio was originally based on $24 \mathrm{~h}$ recording, in which the aim was to estimate the ratio between sympathetic (LF) and parasympathetic (HF) activity; however, there is a disagreement with the LF component. Some studies have suggested that LF is a quantitative indicator of sympathetic modulations; in other studies, LF appears to reflect both sympathetic and parasympathetic activity. Thus, any changes in HR, such as tachycardia, may be result of the preservation of sympathetic and decreased parasympathetic activity, or increased sympathetic and preservation of parasympathetic activity or, simultaneously, increased sympathetic and decreased parasympathetic activity.

The question is to what extent is the LF/HF ratio influenced by the spectral powers LF and HF in our model, although parasympathetic activity is higher in both sexes and in both light periods? The results of our study show that dependence of the LF/HF ratio on LF and HF also depends on sex and on the light periods. LF significantly affected the LF/HF ratio in females but not in males, while the effect of HF on the LF/HF ratio was negligible in both sexes and in both light periods (Table 3). However, these conclusions, in the light of these doubts, should be interpreted with caution.

\section{Conclusions}

Zoletil has a tachycardic effect in both sexes and in both light periods of the rat regimen day. In females, ANS is involved in HR changes in both light periods, while in males, HR exhibited no dependence on ANS activity. Therefore, we speculate that it was predominantly determined by other factors. In females, HRV was determined by sympathetic and baroreflex activity in both light periods, while in males, HRV was determined by parasympathetic

Table 1. Correlation coefficients for spectral powers of HRV, total spectral power of HRV, and HR

\begin{tabular}{|c|c|c|c|c|}
\hline HR (beats/min $-\mathrm{ms}^{2}$ ) & Female, light & Female, dark & Male, light & Male, dark \\
\hline $\mathrm{HF}-\mathrm{HR}$ & $r=-0.76$ & $r=-0.54$ & $\mathrm{r}=-0.23$ & $\mathrm{r}=-0.06$ \\
\hline LF - HR & $r=-0.72$ & $\mathrm{r}=-\mathbf{0 . 5 1}$ & $\mathrm{r}=-0.27$ & $\mathrm{r}=0.12$ \\
\hline VLF - HR & $r=-0.59$ & $\mathrm{r}=-\mathbf{0 . 5 5}$ & $\mathrm{r}=-0.34$ & $\mathrm{r}=0.13$ \\
\hline Total power of HRV - HR & $r=-0.83$ & $r=-0.54$ & $\mathrm{r}=-0.29$ & $\mathrm{r}=-0.06$ \\
\hline
\end{tabular}

Bolded values indicate a statistically significant correlation. HR: Heart rate; VLF: Very-low frequency; LF: Low-frequency; HF: High-frequency; HRV: Heart rate variability. 
Table 2. Correlation coefficients for spectral powers of HRV and total power of HRV

\begin{tabular}{|c|c|c|c|}
\hline ms $^{2}$ & Female, light & Female, dark & Male, light \\
\hline HF, total power of HRV & $\mathbf{r}=\mathbf{0 . 7 3}$ & $\mathbf{r = 0 . 9 0}$, dark & $\mathbf{r = 0 . 9 6}$ \\
\hline LF, total power of HRV & $\mathbf{r = 0 . 9 7}$ & $\mathbf{r = 0 . 9 9}$ & $\mathbf{r = 0 . 5 9}$ \\
\hline VLF, total power of HRV & $\mathbf{r = 0 . 9 2}$ & $\mathbf{r = 0 . 9 8}$ & $\mathbf{r = 0 . 9 5}$ \\
\hline
\end{tabular}

Bolded values indicate a statistically significant correlation. VLF: Very-low frequency; LF: Low-frequency; HF: High-frequency; HRV: Heart rate variability.

Table 3. Correlation coefficients for $\mathrm{LF}$ and $\mathrm{HF}$, and $\mathrm{LF} / \mathrm{HF}$ ratio

\begin{tabular}{|c|c|c|c|c|}
\hline & \multicolumn{2}{|c|}{ Female } & \multicolumn{2}{|c|}{ Male } \\
\hline & Light & Dark & Light & Dark \\
\hline $\mathrm{LF}\left(\mathrm{ms}^{2}\right), \mathrm{LF} / \mathrm{HF}$ & $r=0.90$ & $r=0.99$ & $\mathrm{r}=0.39$ & $\mathrm{r}=0.19$ \\
\hline $\mathrm{HF}\left(\mathrm{ms}^{2}\right), \mathrm{LF} / \mathrm{HF}$ & $\mathrm{r}=0.34$ & $\mathrm{r}=0.29$ & $\mathrm{r}=-0.08$ & $\mathrm{r}=-0.11$ \\
\hline
\end{tabular}

Bolded values indicate a statistically significant correlation. LF: Low frequency; HF: High frequency; LF/HF: Ratio between LF and HF.

Table 4. Summarized results and conclusions

\begin{tabular}{|l|c|c|}
\hline & Females \\
\hline Heart rate & Tachycardia, loss of LD differences & Tachycardia, maintenance of LD differences \\
\hline $\begin{array}{l}\text { Effect of HRV on HR } \\
\text { Light } \\
\text { Dark }\end{array}$ & Yes \\
\hline $\begin{array}{l}\text { Impact of spectral powers on HRV } \\
\text { Light } \\
\text { Dark }\end{array}$ & Yes \\
\hline $\begin{array}{l}\text { Impact of LF, HF on the ratio LF/HF } \\
\text { Light }\end{array}$ & NF and VLF prevail \\
Dark & LF and VLF prevail & HF prevails \\
\hline
\end{tabular}

HRV: Heart rate variability; LD: Light-dark; LF: Low frequency; HF: High frequency; VLF: Very low frequency; LF/HF: Ratio between LF and HF.

activity. LF significantly influenced the LF/HF ratio in females, but not in males, while the effect of HF on the LF/HF ratio was negligible in both sexes and in both light periods. Our conclusions are summarized in the table below (Table 4).

\section{Conflict of interests}

The authors declare that they have no conflict of interests.

\section{References}

1. SvorcJr P, Bačová I, Svorc P, Buzga M (2013) Autonomic nervous system under ketamine/xylazine and pentobarbital anaesthesia in a Wistar rat model: A chronobiological view. Prague Med Rep 114: 72-80. [Crossref]

2. Guzzetti S, Marchi A, Bassani T, Citerio G, Porta A (2015) Univariate and bivariate symbolic analyses of cardiovascular variability differentiate general anesthesia procedures. Physiol Meas 36: 715-726. [Crossref]
3. Meyer MR, Haas E, Barton M (2006) Gender differences of cardiovascular disease: new perspectives for estrogen receptor signaling. Hypertension 47: 1019-1026. [Crossref]

4. Koresh O, Kaplan Z, Zohar J, Matar MA, Geva AB, et al. (2016) Distinctive cardiac autonomic dysfunction following stress exposure in both sexes in an animal model of PTSD. Behav Brain Res 308: 128-142. [Crossref]

5. Molcan L, Teplan M, Vesela A, Zeman M (2013) The long-term effects of phase advance shifts of photoperiod on cardiovascular parameters as measured by radiotelemetry in rats. Physiol Meas 34: 1623-1632. [Crossref]

6. Picker O, Scheeren TWL, Arndt JO (2001) Inhalation anaesthetics increase heart rate by decreasing cardiac vagal activity in dogs. Br J Anaesth 87: 748-754. [Crossref]

7. Yuan H, Silberstein SD (2016) Vagus nerve and vagus nerve stimulation, a comprehensive review: Part III. Headache 56: 479-490. [Crossref]

Copyright: C2020 Svorc P. This is an open-access article distributed under the terms of the Creative Commons Attribution License, which permits unrestricted use, distribution, and reproduction in any medium, provided the original author and source are credited. 